\title{
Work Function Gas Sensors at Room Temperature by means of Conductive Polypyrrole Thin-films
}

\author{
C. R. Zamarreño ${ }^{1}$, P. Davydovskaya ${ }^{2}$, R. Pohle ${ }^{2}$, M. Fleischer ${ }^{2}$, I. R. Matias ${ }^{1}$, F. J. Arregui ${ }^{1}$ \\ ${ }^{1}$ Public University of Navarra, Campus Arrosadia, 31006 Pamplona SPAIN, \\ carlos.ruiz@unavarra.es \\ 2 SIEMENS AG, Otto-Hahn-Ring 6, 81739 Munich GERMANY
}

\begin{abstract}
:
This work presents a first approach to the fabrication of room temperature floating gate GasFETs based on the readout of the work function of conductive polypyrrole (PPy) coatings. PPy coatings have been fabricated onto Au and Pt coated alumina substrates by means of the in-situ polymerization technique. The response of the coatings to different gases and volatile organic compounds (VOCs), such as ethanol, acetone, methylamine or isopropanol and gases has been tested using the Kelvin probe (KP) setup. Results have revealed good sensitivities to isopropanol and methylamine at room temperature. Relative humidity $(\mathrm{RH})$ was found to be a decisive factor, which conditioned the sensitivity of the coatings to VOCs and gases as well as the overall coating response. Additionally, good sensitivity to low weight hydrocarbons was found when the substrate was heated to $180^{\circ} \mathrm{C}$.
\end{abstract}

Key words: work function, polypyrrole, in-situ polymerization, Kelvin probe, relative humidity, volatile organic compounds.

\section{Introduction}

Nowadays, gas sensing applications cover a vast and heterogeneous field from air or combustion monitoring in industrial and domestic environments to the detection of diseases in breath analysis, which requires the accomplishment of specific premises for each case. For instance, high-temperature operated sensors that can provide high stability and fast response in some cases should be discarded for portable wireless long-term operation with batteries or when the operation temperature can derive in degradation and bad operation of the sensor itself [1-2]. Conductive polymers have largely concentrated the attention of the scientific community for the fabrication of gas sensors at room temperature by means of monitoring the change of resistance [3]. Apart from resistance readout there are other properties of conducting polymers that change upon exposure to volatile compounds, such as work function, which is basically based on surface interactions, and can be measured at room temperature [4-5]. This work presents a first approach for the fabrication of ambient temperature floating gate FET devices based on the readout of the work function of conductive polypyrrole (PPy) coatings.

\section{PPy coating Fabrication}

All chemicals were used without further purification. The samples have been fabricated from $\mathrm{Pt}$ and $\mathrm{Au}$ films sputtered onto alumina squares $(0.5 \times 0.5 \mathrm{~cm})$. The samples were washed before testing with ethanol and distilled water and then dried with nitrogen. PPy coatings have been fabricated by means of the in-situ polymerization technique using $\mathrm{FeCl}_{3}$ as dopant as it is described elsewhere [6]. Pyrrole monomer (Py) solution (5mmol) and $\mathrm{FeCl}_{3}$ solution $(10 \mathrm{mmol})$ were prepared separately in $10 \mathrm{ml}$ and $15 \mathrm{ml}$ distilled water respectively. Then, the Py solution was added to the $\mathrm{FeCl}_{3}$ solution and stirred vigorously. The samples were placed afterwards into the solution in vertical position for 5 hours. After the polymerization process, the samples were rinsed thoroughly with distilled water in order to remove the bad adhered material, dried under nitrogen flow and then kept in a desiccator overnight before testing.

\section{Work Function Measurements}

All the measurements were performed at room temperature $\left(23-25^{\circ} \mathrm{C}\right), 40 \%$ relative humidity $(\mathrm{RH})$ and at a constant gas flow (synthetic air as the carrier) of $1 \mathrm{l} / \mathrm{min}$ unless otherwise stated. 
The PPy samples were arranged in a Kelvinprobe (KP) setup [7-8], using a gold grid with a diameter of $3 \mathrm{~mm}$ as the reference electrode. In order to maintain an equal potential $\mathrm{Pt}$ and $\mathrm{Au}$ wires were connected to the Pt and Au layer respectively prior to the PPy coating fabrication and grounded together with the reference gold electrode during the measurement process. Contact potential difference (CPD) was measured at low (ppm) concentrations of different gases, such as NO, ethanol, Acetone, $\mathrm{H}_{2}$, acetaldehyde, methylamine and isopropanol, and compared with the bare $\mathrm{Pt}$ and Au reference samples.

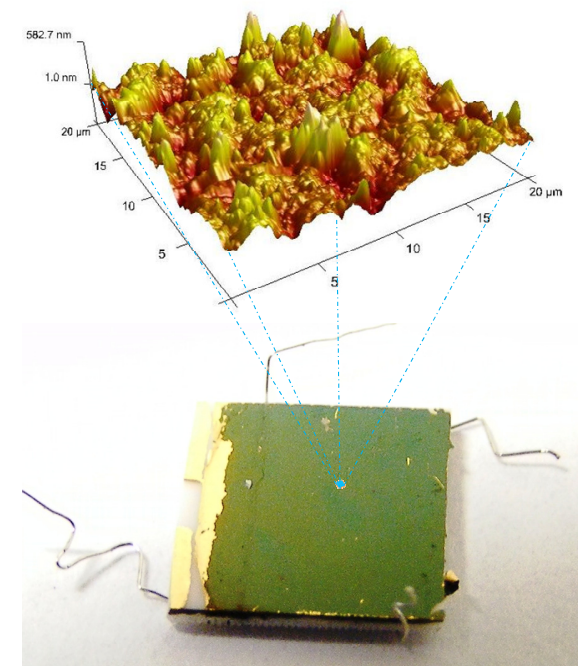

Fig. 1. Top: AFM image of the PPy coating. Bottom: KP sample coated with PPy.

\section{Results and Discussion}

Thin ( 500nm), homogeneous coatings were fabricated onto $\mathrm{Au}$ and $\mathrm{Pt}$ covered alumina substrates as it is represented in Fig. 1. Tapping mode atomic force microscopy analysis (Innova from Veeco Inc.) of the samples revealed an average surface roughness of $\sim 130 \mathrm{~nm}$ (see Fig. 1).

CPD between a gold electrode and the PPy coatings fabricated onto $\mathrm{Au}$ (black solid line) and Pt (red solid line) has been compared with the CPD between a gold electrode and the $A u$ (black dotted line) and $\mathrm{Pt}$ (red dotted line) coated alumina reference respectively. In Fig. 2 it is presented the response of the devices at different concentrations of $\mathrm{NO}(0.02,0.05$ and $0.1 \mathrm{ppm}), \mathrm{H}_{2}(0.02,0.03$ and $0.05 \mathrm{ppm})$, ethanol (5 and $15 \mathrm{ppm}$ ) and acetone (5 and $20 \mathrm{ppm})$. It is important to note that the response to $\mathrm{NO}$ is inverted in comparison with the references, which denotes the influence of the PPy overlayer. The response of the PPy coated samples to ethanol and acetone is similar or even worse to that of the reference samples. Here, the response of the PPy coated samples to ethanol and acetone could be also masked by the long recovery time after the $\mathrm{NO}$ exposure associated to the slope of the solid lines. Additionally, as it happened with the reference samples, there no appreciable response to low $\mathrm{H}_{2}$ concentrations.

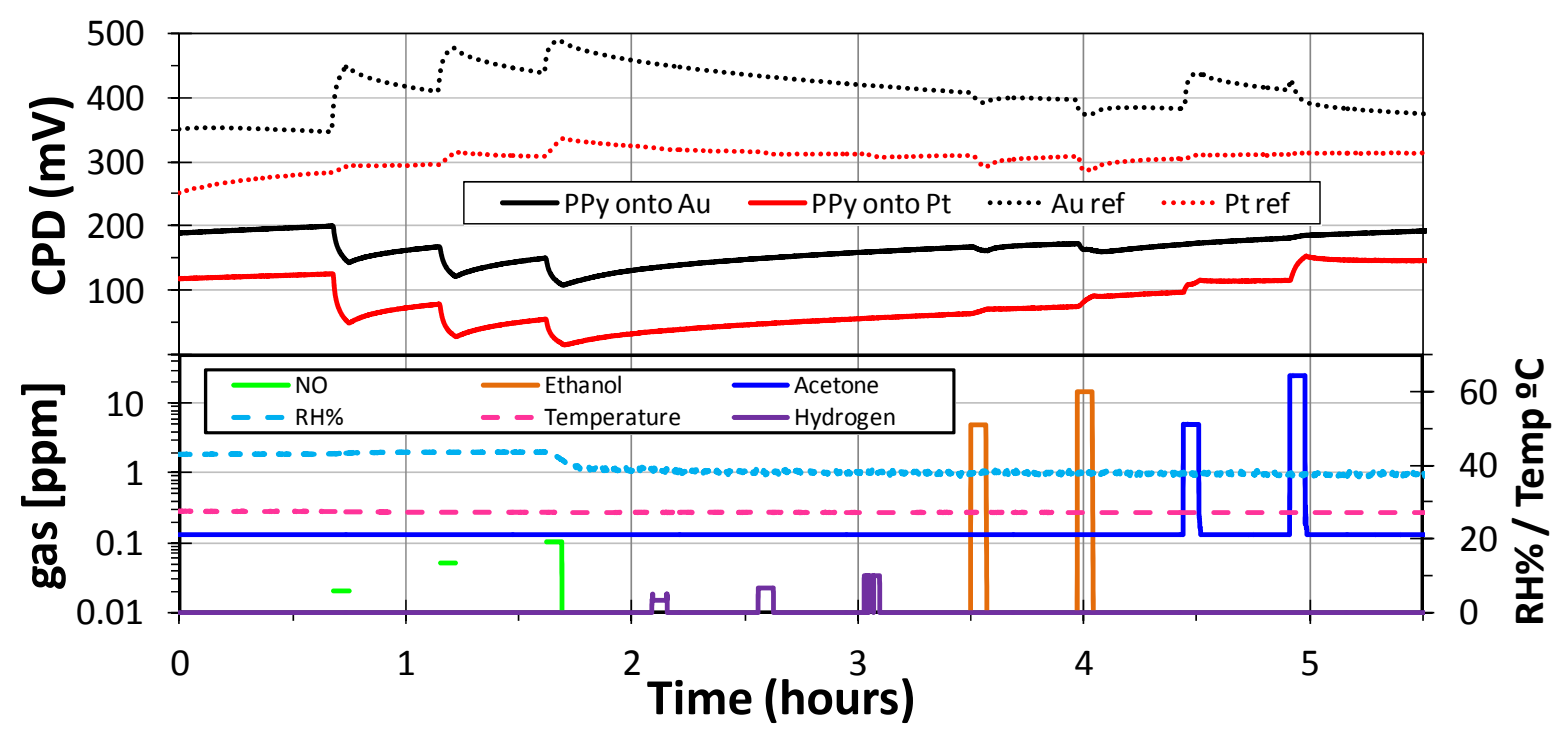

Fig. 2. Kelvin probe measurements of PPy coatings fabricated onto Au and Pt (solid lines) and Au and Pt references (dotted lines) to different gases and concentrations. 


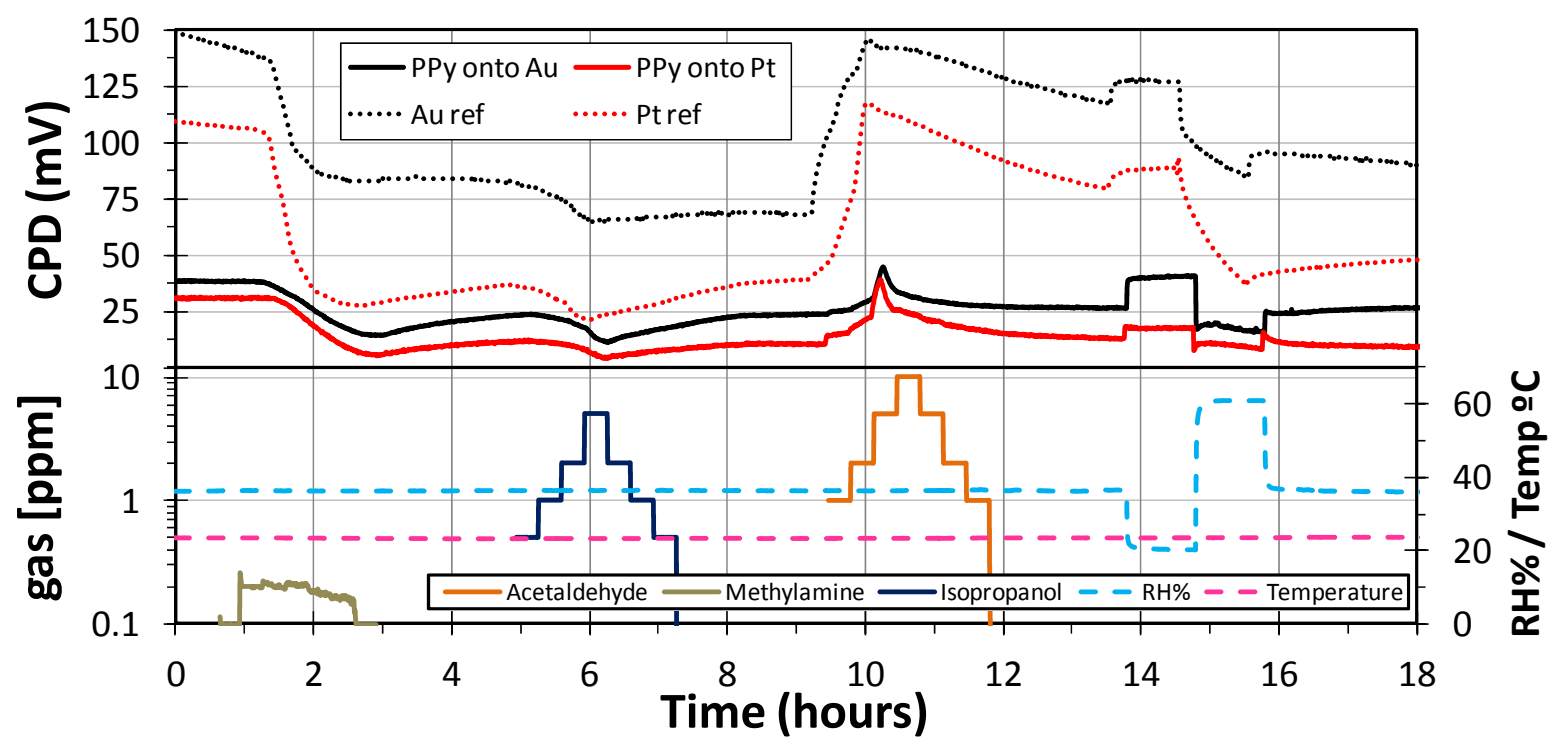

Fig. 3. Kelvin probe measurements of PPy coatings fabricated onto Au and Pt (solid lines) and Au and Pt references (dotted lines) to different gases, concentrations and $R H$.

In Fig. 3 it is presented the response of the devices at different concentrations of methylamine $(0.2 \mathrm{ppm})$, isopropanol (from 1 to $5 \mathrm{ppm}$ ), and acetaldehyde (from 1 to $10 \mathrm{ppm}$ ) as well as the response to different $\mathrm{RH}$ conditions. From Fig. 3 it can be extracted that the CPD variation of the reference samples is greater in magnitude than the variation obtained with the PPy coated samples for methylamine and isopropanol while it is within the same order of magnitude for Adetaldehyde and $\mathrm{RH}$. In contrast, the response time of the PPy coated samples seems to be faster than the response of the bare references in general and it is especially marked in the $\mathrm{RH}$ variation.

Further experiments were performed in order to observe the response to $\mathrm{RH}$ variations with both $\mathrm{Au}$ and Pt PPy coated samples. In Fig 4 $\mathrm{RH}$ variation revealed faster response and recovery times than references (see Figs. 3 and 4) with a dynamic range of $\sim 50 \mathrm{mV}$ with the PPy coating fabricated onto $\mathrm{Pt}$.

\section{Conclusions}

This work has studied the fabrication of PPy thin-films onto $\mathrm{Pt}$ and $\mathrm{Au}$ coated alumina substrates by means of the in-situ polymerisation method. KP tests have been performed with the PPy coated samples in order to study the sensitivity to low concentrations of different gases and VOCs at room temperature. Moreover, PPy coatings have revealed good sensitivity to $\mathrm{RH}$ variations in the range $20-60 \%$. Further studies are needed in order to investigate the coating ageing and saturation as well as to establish the cross-sensitivity of the measures under different conditions ( $\mathrm{RH}$ and Temperature).

\section{Acknowledgements}

This work was funded in part by the Spanish Ministry of Education and Science-FEDER TEC2010-17805/MIC and Government of Navarre research grants.

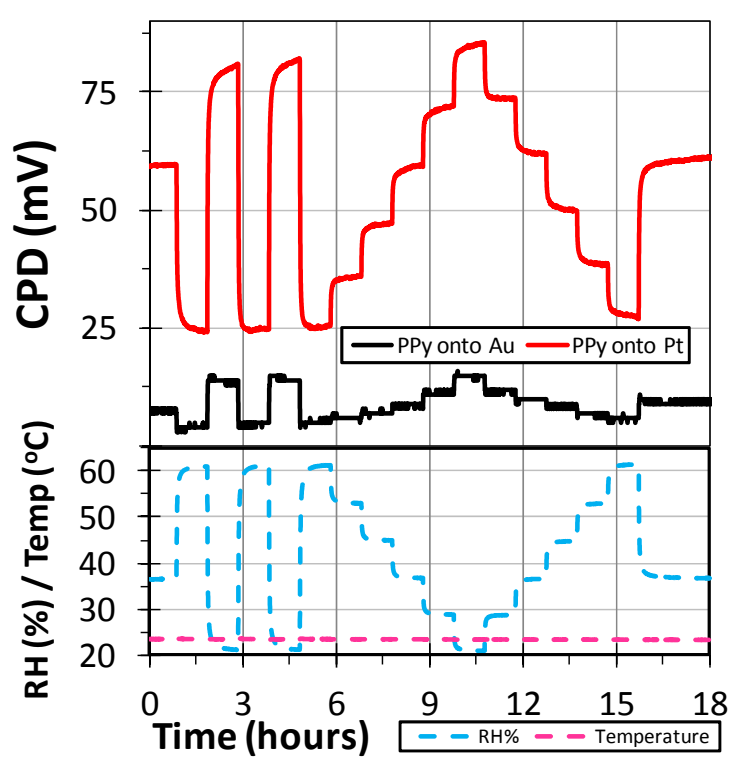

Fig. 4. Kelvin probe measurements of PPy coatings fabricated onto Au (dark line) and Pt (red line) at different $\mathrm{RH} \%$. 


\section{References}

[1] M. Fleischer, Advances in application potential of adsorptive-type solid state gas sensors: Hightemperature semiconducting oxides and ambient temperature GasFET devices, Meas. Sci. Technol. 19, 042001 (2008); doi: 10.1088/09570233/19/4/042001

[2] R. Moos, K. Sahner, M. Fleischer, U. Guth, N. Barsan, U. Weimar, Solid State Gas Sensor Research in Germany - a Status Report, Sensors 9, 4323-4365 (2009); doi:10.3390/s90604323

[3] P. D. Harris, W. M. Arnold, M. K. Andrews, A. C. Partridge, Resistance characteristics of conducting polymer films used in gas sensors, Sensors and Actuators B 42(3), 177-184 (1997); doi:10.1016/S0925-4005(97)80334-6

[4] D. Blackwood, M. Josowicz, Work function and spectroscopic studies of interactions between conducting polymers and organic vapors, Phys. Chem. 95(1), 493-502 (1991); doi:
10.1021/j100154a086
[5] J. V. Hatfield, J. A. Covington, J. W. Gardner, GasFETs incorporating conducting polymers as gate materials, Sensors and Actuators B 65, 253256 (2000); doi: 10.1016/S0925-4005(99)003287

[6] M. Ferenets, A. Harlin, Chemical in situ polymerization of polypyrrole on poly(methyl metacrylate) substrate, Thin Solid Films 515, 5324-5328 (2007); doi: 10.1016/j.tsf.2007.01.008

[7] M. Zimmer, M. Burgmair, K. Scharnagl, A. Karthigeyan, T. Doll, I. Eisele, Gold and platinum as ozone sensitive layer in work-function gas sensors. Sensors and Actuators B 80, 174-178 (2001); doi: 10.1016/S0924-4247(01)00673-2

[8] B. Ostrick, R. Pohle, M. Fleischer, H. Meixner, TiN in Workfunction Type Sensors: A stable ammonia sensitive material for room temperature operation with low humidity cross sensitivity. Sensors and Actuators B 68, 234-239 (2000); doi: 10.1016/S0925-4005(00)00434-2 